Cinémas

Revue d'études cinématographiques

Journal of Film Studies

\title{
Un cinéma zazique?
}

\section{Johanne Bénard}

Volume 4, numéro 3, printemps 1994

URI : https://id.erudit.org/iderudit/1001042ar

DOI : https://doi.org/10.7202/1001042ar

Aller au sommaire du numéro

\section{Éditeur(s)}

Cinémas

\section{ISSN}

1181-6945 (imprimé)

1705-6500 (numérique)

Découvrir la revue

Citer cet article

Bénard, J. (1994). Un cinéma zazique? Cinémas, 4(3), 135-152.

https://doi.org/10.7202/1001042ar

\section{Résumé de l'article}

Cet article veut montrer comment l'étude de l'adaptation cinématographique (par Louis Malle) du roman de Raymond Queneau, Zazie dans le métro, doit tenir compte d'un premier travail de transposition qu'on trouve dans le roman lui-même : le passage de l'oral à l'écrit. C'est à partir de là que l'auteure pose l'hypothèse de l'« intransposabilité » du roman. Hypothèse qu'elle prouve ensuite en considérant les différences fondamentales entre l'énonciation romanesque (déictique) et l'énonciation filmique (réflexive). Elle conclut ainsi que le film de Malle est, somme toute, impuissant à traduire les petites secousses du langage zazique. d'utilisation que vous pouvez consulter en ligne.

https://apropos.erudit.org/fr/usagers/politique-dutilisation/ 


\title{
Un cinéma zazique?
}

\section{Johanne Bénard}

\begin{abstract}
RÉSUMÉ
Cet article veut montrer comment l'étude de l'adaptation cinématographique (par Louis Malle) du roman de Raymond Queneau, Zazie dans le métro, doit tenir compte d'un premier travail de transposition qu'on trouve dans le roman lui-même: le passage de l'oral à l'écrit. C'est à partir de là que l'auteure pose l'hypothèse de l' «intransposabilité" du roman. Hypothèse qu'elle prouve ensuite en considérant les différences fondamentales entre l'énonciation romanesque (déictique) et l'énonciation filmique (réflexive). Elle conclut ainsi que le film de Malle est, somme toute, impuissant à traduire les petites secousses du langage zazique.
\end{abstract}

\begin{abstract}
In order to study Louis Malle's cinematographic adaptation of Raymond Queneau's Zazie dans le métro, one must take into account a prior transposition in the novel itself - the passage from spoken to written word. From this starting point, the author proposes the hypothesis that the novel is "untransposable," a hypothesis that she proceeds to prove by examining the fundamental differences between the novelistic utterance (deictic) and the filmic utterance (reflexive). She concludes that, on the whole, Malle's film is powerless to convey all the ins and outs of Zazie-talk.
\end{abstract}

"Doukipudonktan ». D’emblée le roman de Raymond Queneau, Zazie dans le métro, exhibe son projet: transcription de l'oral et subversion de l'écrit et du genre romanesque. Mais comment lit-on ce texte maintenant, 30 ans plus tard? Le projet de 
transposition de l'oral dans l'écrit ne paraît-il pas lui-même bien daté et, en outre, discrédité par ses accents populistes? Dans le champ littéraire de la seconde moitié du XXe siècle, il apparaît largement dépassé par le projet du nouveau roman, lui toujours vivant ou plutôt semblant toujours prêt à renaître au prix de nombreuses métamorphoses - telle la nouvelle autobiographie. Cette tentative de faire passer la langue orale dans l'écrit du roman semblerait donc avoir plutôt avorté. Elle n'a pas rallié d'adeptes et a été abandonnée par Queneau lui-même, devenu oulipien et poète. La parution de Zazie dans le métro en 1959 est par ailleurs en retard sur les textes théoriques de Queneau concernant la langue parlée, datant eux de 1937 et de 1955 (voir $B \hat{a}$ tons, chiffres et lettres ${ }^{1}$ ); mais, il est vrai, précède de 10 ans le moment où Queneau se rétracte et revient sur sa promotion de la langue parlée (voir son Errata $^{2}$ ). Ensuite, là où le nouveau roman, malgré toutes les réserves et les dénégations de ses praticiens, semble malgré tout constituer ce qu'on pourrait appeler un mouvement littéraire, le roman oralisé (pour lequel on n'a, du reste, pas de nom consacré) n'a été qu'un point de rencontre accessoire entre des écrivains aux parcours par ailleurs très différents. À côté de Queneau, on trouve ainsi Louis-Ferdinand Céline qui, lui, s'en tiendra strictement à son projet de transposition de la langue parlée, voire cherchera à le promouvoir désespérément pour faire oublier ses fautes politiques. Et je rappellerai pour mémoire que Queneau signale en 1937 la réussite stylistique de Voyage au bout de la nuit (paru en 1932), mais que Zazie dans le métro est publié au moment où Céline publie sa trilogie allemande, soit près de 30 ans donc après le Voyage ${ }^{3}$.

Qu'en est-il alors de cette autre transposition que constitue le film de Louis Malle? Si je me suis permise de faire cette mise au point historique sur le roman, c'est que l'étude de la réécriture filmique de Zazie doit tenir compte de ce premier travail du texte - qu'on oublie peut-être maintenant —, qui consiste à faire passer l'oral dans l'écrit. En fait, en présupposant que le projet de Zazie dans le métro est la transposition de l'oral, mon hypothèse de départ est la suivante: l'adaptation de Zazie au cinéma est à strictement parler impossible. Cela relève de l'évidence: le cinéma parlé ne peut qu'effacer ou annihiler le travail 
de transposition du texte. Autrement dit, dans les dialogues du film, on ne sent plus les efforts de l'écriture quenalienne pour coller à l'oral et subvertir le système orthographique. Mais, me dira-t-on, ce travail de transposition de l'oral n'est pas seul dans Zazie. Je me contenterai de répondre pour l'instant qu'il pourrait être emblématique de tout le travail du texte. J'y reviendrai. Qu'on me permette ici de retarder la démonstration... pour considérer une seconde hypothèse concernant l'adaptation du roman, celle-là tout à fait à l'opposé.

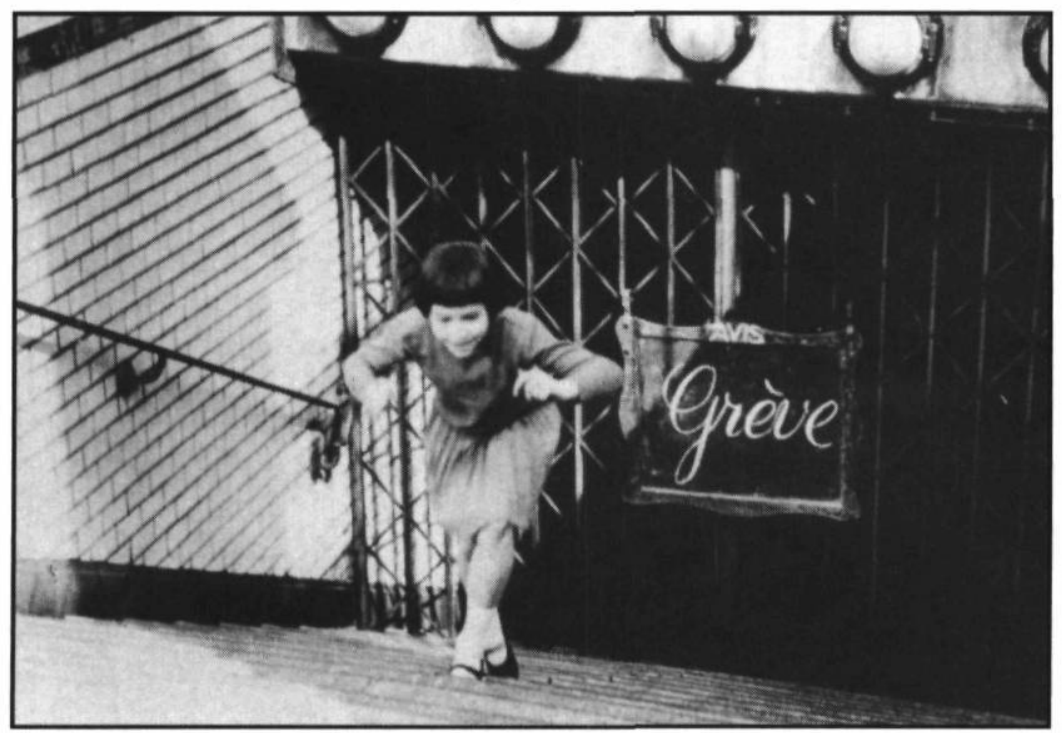

Zazie dans le métro de Louis Malle (1960)

Collection Cinémathèque québécoise

Lorsqu'on lit la critique sur l'adaptation filmique, on est surpris de son uniformité: cette critique s'entend pour dire que l'adaptation de Malle (la même année que le roman) est parfaitement réussie. Le cinéaste a bien transposé (voilà que le terme trouve tout son sens: "faire changer de forme ou de contenu en faisant passer dans un autre domaine", dit le Robert) la langue du roman dans le langage cinématographique. En fait, c'est Malle lui-même qui aurait donné le ton lorsqu'il affirme, dans 
une phrase qui sera elle-même souvent citée dans le discours critique:

Ce qui m'intéresse chez Queneau c'est que toute son œuvre est une critique interne de la littérature: de l'orthographe à la métaphysique, il détruit, par la forme, le fond. En adaptant Zazie dans le métro, J.-P. Rappeneau et moi avons tenté de trouver des équivalences pour arriver à la même critique de l'écriture cinématographique $[\ldots]^{4}$.

Ce que je voudrais ici, donc, c'est faire jouer l'une contre l'autre ces deux hypothèses contraires: l'une (disons la mienne) serait celle de l' "intransposabilité " du roman; l'autre (de Malle reprise ad nauseam par la critique), celle du tour de force d'un film qui est parvenu, contre toute attente - cette deuxième hypothèse se pose donc en quelque sorte comme l'envers de la première —, à faire passer la subversion d'un code à l'autre, en trouvant des "équivalences". Au bout du compte, ce que je veux montrer, ce sont les limites de cette opération de mise en équivalence des structures ou des processus de deux langages: celui du texte et celui du film. Le texte de Queneau ne résiste-t-il pas? ne serait-il pas irréductible?

\section{De l'oral à l'écrit}

Pour éviter les jugements hâtifs sur la (pseudo-)relation d'équivalence entre les énonciations romanesque et filmique, je ne peux faire l'économie d'une analyse textuelle. Je commencerai par les mouvements ou contorsions que doit faire le texte quenalien pour se rapprocher de l'oral. Histoire d'une première transposition.

Bien sûr, on trouve dans le roman de Queneau, à un premier niveau, les modifications orthographiques plus ou moins conventionnelles pour transcrire l'oral: les contractions ("ptit") et les élisions ("t'entends"), qui sont des petites modifications de la graphie qui n'ont rien de très subversif et auxquelles le genre romanesque, au moins depuis le naturalisme, nous a habitués. À l'inverse, il s'agira parfois de l'ajout de consonnes, pour souligner l'assonance: "la ffine efflorescence de la cuisine ffrançouèze" (p. 125). Ou bien de mots déformés par une lettre ou 
une syllabe importune, pour se rapprocher de quelque prononciation: "egzagérer" (p. 42) ou "guidenappeurs" (p. 115).

Mais le procédé le plus intéressant et le plus original du texte quenalien est sans aucun doute sa "tendance agglutinative". Dans son dernier texte, où il fait la promotion de la langue parlée, Battons, chiffres et lettres, Queneau soutient que cette tendance, trop souvent oubliée, est propre au français (parlé) et qu'il faut en reconnaître la richesse créative: "l'un des rares modes de dérivation du français" (p. 81). Or, en voulant mimer l'oral, le texte quenalien réalise, mais sur un tout autre registre (ce n'est pas là sa visée déclarée), d'autres potentialités de la langue: réflexives ou figurales. En fait, l'agglutination se trouve à des échelles différentes: simple suppression de l'apostrophe ("jveux", "faut mdéfendre"), association de deux mots avec une très légère modification orthographique ("Essméfie", p. 14; "Gridougrogne", p. 77) ou bien, de façon spectaculaire, amalgame de plusieurs syntagmes en un seul mot phonétique: le fameux "doukipudonktan" (d'où qu'ils puent donc tant?) inaugural ou, pris au hasard, "skeutadittaleur" (ce que tu as dit tout à l'heure, p. 10), "Singermindépré" (Saint-Germain-des-Prés, p. 29), "Lagoçamilébou" (la gosse a mis les bouts, p. 37), "Ltipstu" (Le type se tut, p. 54), "Iadssa" (Il y a de ça, p. 54).

Ces monstres lexicaux, que le lecteur doit lire à haute voix pour les déchiffrer, Barthes, quant à lui (dans un article de Critique contemporain de la parution du roman), y voit un "caractère d'agression", un "effet baroque" et la tentative de "faire surgir à la place du mot pompeusement enveloppé dans sa robe orthographique, un mot nouveau, indiscret, naturel, c'est-à-dire barbare [...]" (p. 127) ${ }^{5}$. J'irais maintenant plus loin et, dans les termes de la pragmatique, je verrais dans les "coagulations phonétiques" de Zazie (c'est le terme qu'emploie Queneau luimême) des stations autoréflexives privilégiées du texte, où le signe se désigne lui-même et s'opacifie. Là où la langue détourne la visée dénotative du signifié vers le signifiant.

Or, ce phénomène d'opacification est particulièrement intéressant, car à proprement parler paradoxal : alors que le projet de Queneau de transposer l'oral semble aller dans le sens d'une quête de transparence, la langue parlée - on le sait depuis au 
moins Derrida - , se prêtant à tous les mythes de la présence de l'être et de la transparence du langage, le texte de Queneau se retourne sur lui-même et, pourrait-on dire, n'est jamais autant écrit que lorsqu'il veut être oral. La transposition de l'oral est cette utopie d'une coïncidence entre le souvenir de l'oral et un écrit surcodé, chiffré; le référent recule à chaque fois que le texte quenalien crée cet écart, ce vertige, ce vide.

Et ce serait un tel vertige que l'on trouve, plus généralement, dans les calembours, les mots-valises, les pérégrinismes (utilisation de certains éléments linguistiques empruntés à une langue étrangère) qui, eux aussi, bloquent ou retardent le représenté, parce que leur déchiffrement passe nécessairement par un retour sur le signifiant. En fait, les distinctions métalinguistiques ou rhétoriques importent moins ici que ce phénomène d'opacification de la langue que représente, de façon je dirais emblématique, l'oral-écrit des coagulations phonétiques ${ }^{6}$.

Du niveau lexical faut-il alors passer, à l'instar de la méthode linguistique, aux structures syntaxiques: aux phrases nominales, emphatiques, incomplètes et aux erreurs grammaticales qui prêtent aussi au texte quenalien une allure d'oral? Faut-il alors reconnaître les accointances de l'oral et du langage populaire ou de l'argot? C'est là une piste qui m'éloignerait de mon propos parce que, d'une part, l'autoréflexivité de la langue y est moins évidente et parce que, d'autre part, à l'opposé par exemple d'un Céline, ce ne sont pas là les procédés privilégiés d'oralisation du texte quenalien. Je propose donc de faire un saut (qualitatif) et de passer du niveau lexical au niveau de l'énonciation, en sautant le niveau de la phrase. Ce que nous trouvons alors, ce sont ce que j'appellerais les figures de l'énonciation. Tout se passe en fait comme si les transformations phonétiques avaient non seulement des effets de rayonnement dans la phrase (ou plus justement, pour reprendre la métaphore de l'opacification, des effets d'ombre), mais également comme s'ils étaient pris en charge par une énonciation prolifique en figures ironiques ou parodiques. Et c'est donc de ces figures que je voudrais donner une idée avant de passer à l'énonciation du film.

D'un côté, il faut voir comment la véritable subversion du texte quenalien (qui du coup dénature tout projet utopique de 
transposition de l'oral) tient peut-être au fait que les jeux phonétiques se retrouvent indistinctement dans le discours rapporté des personnages et dans celui du narrateur. D'un autre côté, il faut voir comment le narrateur revient sur ces transcriptions, pour en rajouter et s'en moquer. J'en donnerai un exemple puisé dans les premières pages - qui, à l'instar du fameux "Doukipudonktan", programment en quelque sorte une certaine lecture du texte. Il s'agit de la conversation entre Gabriel et le "ptit type": un échange un peu rude parce que "le ptit type" a insulté Gabriel en lui disant qu'il puait :

— Tu pues, eh gorille.

Gabriel soupira. Encore faire appel à la violence. Ça le dégoûtait cette contrainte. Depuis l'hominisation première, ça n'avait jamais arrêté. Mais enfin fallait ce qu'il fallait. C'était pas sa faute à lui, Gabriel, si c'était toujours les faibles qui emmerdaient le monde. Il allait tout de même laisser une chance au moucheron.

- Répète un peu voir, qu'il dit Gabriel.

Un peu étonné que le costaud répliquât, le ptit type prit le temps de fignoler la réponse que voici :

- Répéter un peu quoi?

Pas mécontent de sa formule, le ptit type. Seulement l'armoire à glace insistait: elle se pencha pour proférer cette pentasyllabe monophasée:

- Skeutadittaleur [...] (p. 10).

Ainsi, je dirais que l'intervention du narrateur vient ralentir le dialogue et contrecarre tout l'effet de transparence et de spontanéité que pourraient avoir (et je dis bien "pourraient " à cause du caractère utopique ou illusoire de cette transposition) les reparties elles-mêmes. La désignation de l'amalgame syntagmatique comme d'une "pentasyllabe monophasée" fait donc, par avance, retour sur le signe ("Skeutadittaleur "), déjà autoréflexif; il s'agirait donc d'un retour au second degré: "méta-métalinguistique». D'ailleurs, les niveaux ne finissent plus de s'ajouter, puisque l'expression ironique est elle-même autoréflexive, dans la mesure où le terme "pentasyllabe" paraît déplacé (à cause de sa grandiloquence) et où le terme "monophasée" (qui ne 
s'emploie normalement que pour l'électricité) est un néologisme de sens; s'il ne s'agit pas là de bloquer le signifié, il s'agit tout au moins de le faire dévier.

Par ailleurs, on trouve là tout un jeu au niveau de la chaîne anaphorique. Les désignations changeantes des locuteurs de la conversation accentuent en quelque sorte, jusqu'à sa parodie, les mouvements d'une narration à focalisation multiple, qui affectionne les morceaux en style indirect libre: on passe du point de vue de Gabriel, pour qui l'interlocuteur est le "ptit type" ou le "moucheron" à celui du "ptit type" (on ne dispose pas d'autres désignations plus neutres) pour qui Gabriel est justement "l'armoire à glace». Finalement, il y a le genre de l'anaphore "elle" (qui reprend "l'armoire à glace") : cette reprise anaphorique qui paraît impropre et surprend est un accroc dans la chaîne anaphorique qui, à tout moment donc, refuse de renvoyer de façon transparente à quelque désignation fixe - en l'occurrence au nom propre. Certes, ici, elle est connotativement très riche, puisqu'elle indique et annonce toute l'ambiguïté du sexe de $\mathrm{Ga}$ briel, sur laquelle le roman n'arrêtera pas de jouer.

Partant des jeux phonétiques, on en arriverait donc nécessairement aux jeux proprement déictiques ${ }^{7}$ et, donc, au cœur de l'énonciation quenalienne: irréductible et intransposable.

\section{De l'écrit au film}

J'en viens maintenant à l'adaptation ${ }^{8}$. Si l'on commence par les dialogues, il faut voir d'abord qu'ils ont été repris très souvent tels quels; les élisions, les contractions et les structures syntaxiques qui mimaient l'oral dans le roman ne pouvaient que passer facilement dans les dialogues parlés du cinéma. Le roman fournissait des dialogues tout faits pour le scénario. Or, dans la lignée des films de la Nouvelle Vague et aussi, pourrait-on dire, du théâtre de l'absurde, les comédiens récitent sur un ton tout à fait antinaturel: c'est un jeu le plus souvent outré, avec des changements brusques d'intonation ou d'attitude, qui défie toute vraisemblance psychologique. Parfois même, les personnages semblent s'adresser au spectateur. C'est surtout le cas pour les monologues de Gabriel, comme le monologue inaugural sur le quai de la gare (transposition du monologue intérieur du 
roman) ou le long monologue du haut de la tour Eiffel ${ }^{9}$. Le ton théâtral - comme on le dit, dans le contexte du cinéma, pour parler d'une récitation affectée - et les quelques adresses au spectateur, viendraient ainsi connoter l'artificialité du dialogue, par-delà ses efforts pour coller à la langue parlée. Par-delà cette mimésis ou à cause d'elle? En fait, il me faut l'admettre, les dialogues du film rendent bien la tension des dialogues du roman: combinant effets d'oralité et effets du trop-écrit, ou plus exactement de l'écrit se désignant comme écrit. De même, la grande variété des accents (légèrement germanisants chez Albertine ${ }^{10}$, populaires chez Gridoux, et franchement italiens chez Trouscaillon) a plutôt l'heur d'attirer l'attention sur le matériau sonore lui-même, en opacifiant légèrement le signe.

Mais qu'en est-il du contexte audiovisuel dans lequel sont pris ces dialogues; qu'en est-il de l'ensemble de l'énonciation du film Zazie dans le métro? D'ailleurs, peut-on même parler d'énonciation au cinéma; les concepts de la linguistique conviennent-ils au film, qui n'est un texte qu'en partie linguistique et qui a le propre de combiner plusieurs langages ou plusieurs codes? Alors qu'il ne va pas de soi d'emprunter des concepts à la linguistique pour le texte littéraire (ce qui est, certes, une tout autre question), cela devient de toute évidence particulièrement délicat pour un objet non linguistique, ou plutôt un objet qui n'est pas exclusivement linguistique. Cette question pourrait être la pierre de touche de toute théorie de l' "adaptation" ou de la réécriture filmique - pour laquelle nous n'avons du reste pas de nom. Et elle mérite qu'on s'y arrête un moment avant de comparer les énonciations de Zazie. Je propose donc de faire un petit détour en considérant le dernier livre de Christian Metz: L'Énonciation impersonnelle ou le site du film. Ce livre présente selon moi un bon état présent de la question. Ainsi, selon Metz, il faut, pour parler d'énonciation au cinéma, " concevoir un appareil énonciatif qui ne soit pas essentiellement déictique (et donc anthropomorphique), pas personnel (comme les pronoms que l'on appelle ainsi), et qui n'imite pas de trop près tel ou tel dispositif linguistique [...]" (p. 19). Pas de sujets aux deux bouts de l'énoncé filmique et encore moins d'échange, de cette réversibilité qui fonde la théorie des pronoms chez Benveniste. Les 
marques de l'énonciation filmique seraient réflexives plutôt que déictiques:

Le film nous parle de lui-même, ou du cinéma, ou de la position du spectateur, et c'est alors que se manifeste cette sorte de dédoublement de l'énoncé qui, dans toutes les théories, constitue ce sans quoi on ne pourrait même pas penser à une énonciation (p. $20^{11}$ ).

Autrement dit, pour en revenir au sujet qui nous intéresse, l'adaptation serait le passage d'une énonciation qui se marque de façon déictique à une énonciation qui se marque de façon réflexive. Certes, il faudrait nuancer cette affirmation pour autant que la deixis a aussi, dans l'énonciation, une valeur réflexive. Ainsi, Metz lui-même remarque que la pragmatique tend maintenant plutôt à montrer la réflexivité générale de la langue, de laquelle participe les déictiques ${ }^{12}$. Il reste tout de même que le propre de l'énonciation filmique, qui se réfléchit aussi dans son énoncé, est justement de se passer de déictiques.

Or, il s'avère que les différentes formes par lesquelles l'énonciation se dédouble sont légion dans le film de Malle. Il serait même impensable pour moi, ici, d'en faire un relevé tant soit peu exhaustif. Voyons d'abord ce qui serait la forme la plus importante de ce dédoublement: les longues séquences muettes, dans la tradition du burlesque américain (silent slapstick farce). Il s'agit d'ailleurs en quelque sorte d'un lieu commun de la critique, qui a bien reconnu que ces fragments étaient une façon de rendre le spectateur conscient du film. Je distinguerai d'abord les deux séquences proprement muettes de poursuite, tournées en accéléré: d'abord, la première fugue de Zazie, poursuivie par Turandot, puis la séquence (plus longue) de la poursuite de $\mathrm{Za}$ zie par Pédro - le personnage qui empruntera différents rôles et différents noms dans le roman comme dans le film: Pédro (le satyre), Trouscaillon (le faux flic), Bertin Poirée (l'inspecteur), puis finalement Aroun Arachide (un militaire rappelant Mussolini dans le film). Le topos de la poursuite permet le déballement de tous les procédés burlesques: bousculades, quiproquos avec des passants qui entravent le chemin des poursuivants, trucs loufoques de Zazie pour déjouer, tromper, distraire son adver- 
saire, avec l'apparition et la disparition fortuites de différents accessoires (pétard, miroir, mannequin, appareil-photo, gant de boxe, aimant, etc.), de même que l'insertion de cartons de bande dessinée (pour l'onomatopée "Boum»). Pourquoi devrait-on y voir les plus grands replis du film de Malle? Ces films seconds se démarquent du film principal de différentes manières: non seulement à cause des contrastes (parlé/muet; accéléré/vitesse normale; gags du burlesque / comédie de langage ou de situation), mais aussi parce qu'il s'agit d'une référence à d'autres films - et que ces films burlesques, qui ne paraissent plus maintenant naturels ni vraisemblables, montrent eux-mêmes leur dispositif.

Par ailleurs, on trouve dans le film d'autres séquences de poursuite que seule la bande sonore nous empêche de qualifier de muettes. Deux séquences ont lieu dans les rues encombrées de Paris - à cause de la grève du métro. Il s'agit d'abord de la scène où la veuve Mouaque, Trouscaillon et Zazie poursuivent Gabriel, qui s'est fait enlever par les touristes du car de Fédor Balanovitch. Puis, de la course d'Albertine en vélomoteur (course contre la montre, celle-là) pour aller porter la robe à Gabriel. Plus précisément, nous trouvons là une alternance de séquences qui montrent les différents personnages convergeant vers le cabaret de Gabriel. Ce qui donne le rythme frénétique à ces séquences, alors, c'est tant le contenu de certains plans, parfois tournés en accéléré (la course folle d'Albertine, elle-même poursuivie par Trouscaillon, lui-même poursuivi par la veuve Mouaque; la course du taxi de Charles comme celle du car; la promenade de Zazie somnambule à Pigalle, parmi la foule) que l'alternance rapide des plans eux-mêmes - dans lesquels sont intercalés des plans déroutants où posent les personnages principaux: dans la berceuse de Gabriel ou en farandole, autour d'une fontaine.

Puis finalement, on pourrait classer dans cette catégorie élargie du burlesque (muet ou parlant), la dernière scène de bagarre dans la brasserie, que provoque une altercation entre une touriste et un serveur. Cette bagarre qui oppose le groupe de Gabriel aux serveurs devient vite hors de proportion et conduit à toute une séquence proprement burlesque où la même douzaine 
de serveurs revient inlassablement, de manière invraisemblable, pour se faire attaquer. Cela compose par moments une sorte de ballet comique et mécanique: ainsi, des bouteilles qui assomment les serveurs en file, dans un rythme régulier, ou des assiettes de choucroute, remplies à la chaîne avant de devenir les projectiles qui se substituent aux traditionnelles tartes à la crème. Ou bien, c'est le décor lui-même de la brasserie qui est détruit pour être décomposé en autant de projectiles, alors qu'il révèle un décor plus ancien (de style 1900). Autant dire bien sûr que le décor, réflexivement, se désigne lui-même alors comme décor. D'ailleurs, le film n'a pas fini de connoter le faux et de se redoubler, puisque la finale de cette dernière séquence burlesque, avec un Aroun Arachide en "chemise noire", est l'occasion de la projection, derrière la vitrine de la brasserie, d'un autre film (pour représenter sa troupe) avec bruits d'applaudissements off de foule, de même que d'une seconde destruction du décor, dévoilant cette fois les murs nus du studio - en même temps que l'équipe du tournage.

Cette dernière séquence de cohue, où les marques réflexives se produisent en cascade, permettrait donc au film de se retourner d'une manière indubitable, n'eût été, selon moi, de l'accélération frénétique du film et d'un effet de saturation, qui a plutôt l'heur d'embrouiller complètement le spectateur. Les allures chaotiques et destructrices de cette séquence nous font nous demander si les redoublements énonciatifs n'ont pas des limites et si leur excès ne risque pas d'annihiler l'énonciation elle-même, c'est-à-dire d'empêcher que se raconte (se montre et se dise) une histoire. Le moins que l'on puisse dire est que le spectateur en sort complètement étourdi : somme toute moins attentif aux plissures du film que pressé d'en finir.

Je passerai vite sur tous ces autres petits replis de l'énonciation ou, pour continuer à le dire avec Metz, de tous "ces sursauts du film qui tout à coup se désigne [...] et à tous les sens du mot, se reprend" (p. 165-166) ${ }^{13}$. Les miroirs, les vitrines, les écrans seconds, les surcadrages ou les fréquentes références à d'autres films (au détour d'un plan, d'une réplique ou d'une musique ${ }^{14}$ ) ne sont que de bien légers redoublements en regard de ces longues mises en abyme burlesques. De même pour les 
moments où la caméra se révèle par des mouvements brusques, répétitifs ou imprévus (lorsqu'elle tourne en rond dans la scène de la rencontre entre Trouscaillon et Albertine par exemple) ou par la multiplication des cadrages sur un même objet (comme pour le début de la séquence de la tour Eiffel). De même pour les éclairages surréalistes (la scène de la conversation entre Albertine et Mado Petits-Pieds, où les visages passent du mauve au violet) ou les mauvais raccords du script, qui rendent perceptible le montage des plans.

Me semblent plus intéressants les changements incongrus de plans et les jeux de l'espace. Dans la séquence du quai au début (mais cela arrive aussi dans d'autres séquences), le travelling sur Gabriel, qui remonte la file des passagers, se termine de façon tout à fait déconcertante sur le même passager - facilement reconnaissable parce qu'il lit une revue: Diogène. De deux choses l'une. Ou bien le mouvement de caméra paraît truqué ou bien l'on présuppose que le passager a bougé - et, puisqu'il a dû passer derrière la caméra, il s'agit en quelque sorte d'une "manifestation" du hors-champ. Ailleurs, un raccord astucieux de plans donnera l'illusion qu'une scène de la rue se poursuit dans l'appartement de Gabriel; il s'agit de la présentation, par Zazie, de Pédro à Gabriel. Et puis, ce sont les fameuses scènes de la visite de Paris (dans le taxi de Charles ou le car de Fédor), qui tournent toujours autour de la même église, alors que les personnages croient reconnaître des monuments différents; ce n'est jamais le bon, puisqu'il s'agit de l'église Saint-Vincent-de-Paul, près de la gare du Nord ${ }^{15}$. Or, truquer et limiter l'espace (et l'on pense peut-être aux jeux de perspective des illustrations d'Escher), c'est d'abord, dans ce film, une façon de figurer le piétinement, le sur-place, le circulaire. Laccélération qui domine tout le film (à l'intérieur de certaines séquences comme au niveau de l'ensemble du film, qui aboutit à la frénésie de la dernière bagarre) a comme contrepartie l'immobilité de l'espace, ou plus exactement, parce qu'il faut bien que le film se meuve et avance, les parcours circulaires ou répétés des personnages et / ou de la caméra. Dans les scènes de poursuite, du reste, il arrive souvent que les personnages, littéralement, courent sans avancer ou en faisant des cercles. Et il faut mentionner la présence des "permanents" 
(c'est le scénario qui les désigne ainsi, mais le terme est particulièrement éloquent quant à cette figure du retour du même) qui jouent différents rôles secondaires dans différentes séquences; ce sont eux que l'on trouve par exemple sur le quai au tout début du film.

Enfin, au niveau de la bande sonore, on notera la synchronisation délibérément mauvaise, avec certains effets cocasses (par exemple, quand Zazie pleure devant le métro) ou donnant lieu à des associations incongrues, quand les sons ne correspondent pas à l'image (comme pour les objets au marché). Quant à la parole, j'ajouterais qu'elle est très souvent couverte par d'autres bruits ou perdue dans quelque cacophonie. Et Dieu sait si le bruit (la musique aussi, bien sûr, mais c'est là une tout autre question) est présent dans ce film: tintamarre des scènes de bar et de bagarre, cacophonie des scènes de rue avec les musiciens de l'Armée du Salut (les permanents), rumeur polyglotte du car des touristes. Il arrive même que telle réplique de Zazie devienne tout à coup incompréhensible, parce que la bande sonore est jouée en accéléré ou à reculons. En se décollant de l'image, en s'emballant, en révélant l'artificialité de sa production, c'est toute la bande sonore, bruit et parole, qui constitue aussi un repli énonciatif.

\section{"Causer» n'est pas filmer}

"Tu causes, tu causes, c'est tout ce que tu sais faire", répète le perroquet Laverdure dans le film comme dans le roman. Mais, alors que cette phrase métaphorise la réflexivité ou la performativité de la langue quenalienne (le "causer" comme un faire plutôt qu'un représenter), elle n'a plus la même valeur emblématique dans le film, qui a autre chose à faire que de "causer" et dont les répétitions ne sont pas celles d'un perroquet ou d'une langue qui se retourne sur elle-même, mais celle de tout un film qui, pour montrer son énonciation, n'a d'autres choix (mais les possibilités, certes, sont nombreuses) que de se désigner comme film - et non comme parole. Si l'on veut parler d'équivalences entre le texte écrit et le texte filmé, cela ne peut être qu'à une très grande échelle et non de terme à terme. Et si l'on veut parler de réflexivité de l'énonciation dans les deux cas, il faut bien 
préciser que ces réflexivités sont d'ordre différent et, surtout, ont des effets différents. Autrement dit, la façon dont le film se désigne lui-même ne se rapproche ni de la réflexivité des coagulations phonétiques, là où le signe se retourne sur lui-même, ni de tout le procès de l'énonciation (s'autodésignant)... dans lequel sont pris ces signes opacifiés; là où les jeux sont proprement déictiques.

Et je voudrais revenir ici sur les jeux du nom propre ${ }^{16}$ qui, alors qu'ils sont particulièrement importants dans l'énonciation parodique du roman, ne peuvent être transposés dans le film. En fait, le film ne peut.rendre que la dimension thématique des transgressions identitaires: l'instabilité des oppositions sexuelles (soit le couple Gabriel / Albertine ou Marceline) ou la précarité des rôles sociaux des personnages (soit les différents rôles de Trouscaillon) ${ }^{17}$. Certaines séquences, ainsi, deviennent symboliques: le dédoublement du personnage de Trouscaillon au marché (le même comédien jouant le vendeur et l'acheteur) et la conversation entre Gridoux et Trouscaillon, au sujet de l'absence de nom de ce dernier - alors qu'au moment où Gridoux dit son nom, pour prouver à Trouscaillon que lui le "connaît par cœur ", il devient un homme noir. Le film ajoute ses propres figures réflexives, incapable de rendre compte (sauf bien sûr dans les dialogues) des fluctuations de la chaîne anaphorique qui, par ailleurs, parcourent tout le roman - ces variations n'épargnant pas les pseudo-désignations fixes que sont les noms propres. Quant au changement final d'Albertine en Albert, renversement qui fait écho à l'indétermination du sexe de Gabriel, le film l'annonce, le prépare, le donne à voir progressivement, puisqu'on voit Albertine s'habiller en aviateur dans la loge de Gabriel en conversant distraitement avec Pédro-Trouscaillon-Bertin Poirée. Le personnage du film se dédouble, se déguise, change de rôle, de visage, comme de nom, alors que dans le texte l'instabilité de la chaîne anaphorique est seule, est première et ne représente pas tant l'instabilité de l'identité du personnage qu'elle la produit et s'y réduit. Autrement dit, dans le roman, ce n'est pas le personnage qui change de nom, c'est l'énonciation qui change de désignations et déstabilise toute sa chaîne anaphorique. Et, pour en revenir à la transformation d'Albertine en Albert, là où 
le texte ménageait une place pour un renversement final par le relais d'anaphores circonstancielles ("le manipulateur du montecharge", puis "le lampadophore ${ }^{18}$ "), qui maintenaient en quelque sorte l'indécision, le film tranche et montre la transformation d'un personnage ${ }^{19}$.

Certes, la critique, après avoir vanté les mérites de l'adaptation, admet bien l'impossibilité pour le film de tout transposer. Ainsi, W. D. Redfern, dans son livre d'introduction à Zazie dans le métro pour les étudiants anglophones, soutient que certaines blagues ne peuvent être traduites en langage cinématographique $^{20}$. Or, voilà l'une des phrases qu'il cite:

Elles entendaient, au loin, dans les rues, les pneus se dégonfler dans la nuit.

Redfern sort la phrase de tout son contexte (énonciatif) et ne peut ainsi rendre compte de toute la subtilité de ses jeux avec le sens. Je donne ce contexte:

Là-dessus, elles [Madeleine et Marceline] demeurèrent silencieuses, penseuses, rêveuses. Le temps coulait pas vite entre elles deux. Elles entendaient au loin, dans les rues, les pneus se dégonfler lentement dans la nuit. Par la fenêtre ouverte, elles voyaient la lune scintiller sur le gril d'une antenne de tévé en ne faisant que très peu de bruit (p. 143).

Or, en passant vite sur les légers déplacements qu'occasionne l'intrusion de la syntaxe de la langue parlée (absence du "pas"; "entre elles deux») ou de l'orthographe phonétique (tévé) du parlé, je voudrais m'arrêter sur ce jeu entre le propre et le figuré pour cette image des pneus qui se dégonflent - mais cela vaut aussi pour celle de la lune sur le gril d'une antenne de télévision. Le contexte invite à prendre la phrase comme une figure hyperbolique pour exprimer le temps qui passe lentement et le silence entre les deux femmes, mais le caractère imprévu de l'association des mots (et on pense ici aux images surréalistes) de même que la tendance autonymique propre au langage - et plus particulièrement à l'oral-écrit du texte quenalien — tirent l'énoncé du côté du sens littéral. C'est cette vibration, riche en effets ironi- 
ques dans le texte de Queneau, que ne peut rendre le cinéma à moins d'avoir recours bien sûr à l'énonciation linguistique et à la voix off.

Pour bien saisir la différence des énonciations, on peut voir ainsi comment c'est une phrase paradoxale comme "[Zazie] file droit devant elle en zigzag" (p. 56) qui donne lieu à la longue digression burlesque d'une scène de poursuite. Mais là aussi je donnerai le contexte:

Brusquement, elle [Zazie] se lève, s'empare du paquet et se carapate. Elle se jette dans la foule, se glisse entre les gens et les éventaires, file droit devant elle en zigzag, puis vire sec tantôt à droite, tantôt à gauche, elle court puis elle marche, se hâte puis ralentit, reprend le petit trot, fait des tours et des détours (p. 56-57).

C'est donc que le film, ne pouvant que raplatir l'épaisseur de la langue - qui se mesurerait en quelque sorte par la distance entre le signifiant et le signifié ou entre le littéral et le figuré se contente de représenter les trajets zigzagants, sans la contradiction linguistique de la ligne droite zigzagante, qui tend à annihiler tout renvoi à quelque référent et institue une fracture entre les deux faces du signe. Et, alors, il ne peut compenser que par d'autres effets de miroirs et de replis, révélant moins la matérialité de l'image-son que son artificialité, son caractère fabriqué, ses conventions.

En conclusion, je voudrais proposer une autre façon de considérer les séquences muettes du film de Malle. N'est-ce pas en dernière instance (par-delà tous les jeux de l'énonciation filmique qu'elles permettent) une façon pour le film d'avouer l'impossibilité d'un passage, sans perte ni reste, d'une énonciation à l'autre? Dans ces séquences où la parole s'absente, le film avoue qu'il ne "dit" pas le texte quenalien. Toutes les traces de l'oralisation, qui sont dans ce texte la pierre de touche du travail poétique, comme toutes les traces (déictiques) de l'énonciation, sont en quelque sorte cachées par les replis du film.

Quant au métro, il me semble constituer un autre symptôme, par le biais de l'intertexte des Entretiens avec le Professeur $Y$ (1955) de l'intransposabilité du roman. On sait que, dans la poétique célinienne, le métro est une métaphore privilégiée pour 
rendre compte du travail de transposition de l'oral dans l'écrit. Le style qui se désigne comme un "métro émotif" va droit à l'émotion de la langue parlée. Dans les Entretiens, le (méta)discours sur le métro émotif a comme contrepartie l'histoire de l'interviewer (le Professeur Y) et de l'interviewé (l'auteur), qui doivent aller rejoindre l'éditeur (Gallimard) en traversant, en taxi plutôt qu'en métro, un Paris dont les rues, pleines d'embûches et d'obstacles, les empêchent d'avancer. Or, le film de Malle (à partir du roman) ne pouvait pas mieux rendre "l'encombrement de la surface", contre lequel se définit le style célinien. Toutes ces scènes où on voit les rues de Paris débordant de voitures qui avancent difficilement ou qui sont prises dans d'interminables bouchons de circulation seraient une autre façon de suggérer l'impossibilité, ou tout au moins les paradoxes, de la double transposition: oral / écrit et écrit / film. Mais on pourrait, certes, faire jouer autrement la métaphore du métro, en opposant la poétique célinienne à la poétique quenalienne - malgré leur projet commun de transposer l'oral. À la différence de la quête émotive du style célinien (remontant aux sources de la langue-mère), les détours, les zigzags et les piétinements sont bien le propre des "exercices de style" de Queneau, c'est-à-dire d'un texte tout en surface, tendant à nous faire oublier les profondeurs mythiques du signifié. Le film et ses replis sont impuissants à traduire ces petites secousses du langage zazique, cette façon qu'il a de décoller les deux faces du signe. La réflexivité qui passe d'une énonciation à l'autre ne peut rendre compte de ces parcours impossibles du texte de Queneau, donnant l'impression, comme dans un ruban de Mœbius, que les deux faces du signe sont sur des plans différents. La répétition infinie de ce trajet à l'intérieur de la langue a somme toute peu à voir avec le piétinement et les redoublements du film. Comme le métro, le texte s'est mis en grève... pour que le film puisse avancer. Le revers de la frénésie du film de Malle, c'est bien ce ralentissement qu'opère l'énonciation, dans le livre de Queneau, de la vitesse du signifiant et de celle du signifié, pour tenter de les détacher.

Université Queen’s 